\title{
Conditional Variables Double Sampling Plan for Weibull Distributed Lifetimes under Sudden Death Testing
}

\author{
S. Balamurali and J. Subramani
}

\begin{abstract}
In this paper, we propose a conditional sampling plan called conditional double sampling plan for lot acceptance of parts whose life time follows a Weibull distribution with known shape parameter under sudden death testing. A table is also developed for the selection and application of optimal parameters of the proposed plan for specified two points on the operating characteristic curve namely the acceptable reliability level and the limiting reliability level along with the producer and consumer's risks. The optimization problem is formulated as a nonlinear programming where the objective function to be minimized is the average group number and the constraints are related to lot acceptance probabilities at acceptable reliability level and limiting reliability level under the operating characteristic curve .
\end{abstract}

Keywords--- Acceptable Reliability Level, Average Group number, Conditional Sampling Plans, Limiting Reliability Level, Lot Acceptance, Operating Characteristic Curve, Weibull Distribution

\section{INTRODUCTION}

A CCEPTANCE sampling is one of the major areas of statistical quality control. Acceptance sampling is the methodology that deals with procedures by which decision to accept or reject the lot based on the results of the inspection of samples. Acceptance sampling prescribes a procedure that, if applied to a series of lots, will give a specified risk of accepting lots of given quality. In other words, acceptance sampling yields quality assurance. Implementation of acceptance sampling in industries through the operation of sampling plan yields quality assurance. Use of acceptance sampling is essential to secure ISO certification which gives a passport for larger exports.

One of the major areas of acceptance sampling is variables sampling plans. The main advantage of a variables sampling plan is that it provides better operating characteristics as compared to an attributes sampling plan. In other words, a

Dr. S. Balamurali, Professor and Head, Department of Computer Applications, Kalasalingam University, Krishnankoil 626126, India, E-mail: sbmurali@gmail.com

Dr. J. Subramani, Associate Professor, Department of Statistics, Pondicherry University, Puducherry 605014, India, E-mail: drjsubramani@yahoo.co.in

DOI: 10.9756/BIJDM.1343 variables sampling plan may require smaller sample size than an attributes sampling plan at the specified quality level. Although the attributes sampling incurs lower cost to conduct an experiment, the variables sampling plan with a smaller sample size may lead to the reduced total experiment cost particularly when the inspection is destructive. The variables sampling plan is based on the measurement data, which usually provides more information about the items under inspection than simply labeling the items as conforming or non-conforming.

Several authors have investigated the efficiency of the variables sampling plans and subsequently developed certain efficient variables sampling plans. Pearn and $\mathrm{Wu}$ [11] pointed out that a well-designed sampling plan can minimize the gap between the required and the actual supplied product quality. Hamaker [8] developed the designing methodology of the variables single sampling plan and Sommers [13] proposed a two-point method for determining parameters of a variables double sampling plan. Pearn and $\mathrm{Wu}[11,12]$ considered the design of variables single sampling plan based on the process capability indices, where the plan parameters are obtained by deriving the exact sampling distributions. Variables repetitive group sampling (VRGS) plans were proposed by Balamurali et al. [2] and Balamurali and Jun [3], where they were demonstrated to outperform the single and the double sampling plans in terms of the average sample number. Jun et al. [9] developed single and double variables sampling plans under sudden death testing based on Weibull distribution. Govindaraju and Balamurali [7] extended the concept of chain sampling to variables inspection for a normally distributed quality characteristics. Balamurali and Jun [4] developed multiple-dependent sampling plan for variables inspection. Balamuali and Jun [5] developed a designing methodology to determine the parameters of tightened-normal-tightened (TNT) sampling scheme under variables sampling.

Hence, this paper proposes the designing methodology for determining the parameters of a conditional variables double sampling plan for Weibull distributed life times under sudden death testing. Quick switching system for normally distributed quality characteristics having lower and upper specification limits. The optimal parameters of the proposed plan can be determined for specified two points namely acceptable reliability level (ARL) and lot tolerance reliability level (LTRL) along with the respective risks on the operating characteristic (OC) curve approach. 


\section{SudDen DeATH Testing}

In the reliability sampling, two types of censoring namely time censoring (or type I censoring) and failure censoring (or type II censoring) are applied to reduce the test time of an experiment. Sudden death testing is also frequently applied to life testing problems to reduce the testing time. The total number of items to be tested say $\mathrm{n}$ items is divided into equal sized groups according to the available number of experimental testers (For further details one can refer (Pauscal and Meeker [10] and Vlcek et al. [14]. Thus there are $r$ items in each of $g$ groups so that $n=r g$. The items in each group are tested simultaneously and identically on different testers. The testing of first group of items is run until the first failure occurs. At this juncture, surviving items are suspended and removed from testing. Another equal set of $r$ items is tested next until the first failure is observed. This process is continued until at least one failure is observed from each of $\mathrm{g}$ groups. At the end, $g$ failures are observed from $g$ groups and (r-1)g items are suspended and removed from the testing. Balasooriya [6] developed reliability sampling plans for this type of testing for two parameter exponential distribution. $\mathrm{Wu}$ et al. [15] use the terminology 'limited failure-censored life test' for this type of testing and they have also analyzed the average testing time.

\section{Conditional Double SAMPling Plan}

The concept of conditional double sampling was developed by Baker and Brobst [1]. The conditional double sampling plan has OC curves identical to comparable double sampling plan, but is operationally different since the results of the second sample, if required, are obtained from a related lot rather than the current lot. The operation of conditional double sampling plan is as follows.

- From the lot i, draw a random sample of size $n=r g$ and allocate $r$ parts to each of $g$ groups.

- Perform sudden death testing and observe $Y_{j}$, the time to the first failure from the $\mathrm{j}^{\text {th }}$ group $(\mathrm{j}=1,2, \ldots, \mathrm{g})$. Then calculate the quantity $v_{i}=\sum_{j=1}^{g} Y_{j}^{m}$.

- Accept the lot if $v_{i} \geq k_{a} L^{m}$ and reject the lot if $v_{i}<k_{r} L^{m}$. If $k_{r} L^{m} \leq v_{i}<k_{a} L^{m}$ then defer the decision until the results of the lot $(i+1)$ are obtained.

- From the lot $(i+1)$, draw a random sample of size $\mathrm{n}=\mathrm{rg}$ and allocate $\mathrm{r}$ parts to each of $\mathrm{g}$ groups.

- Perform sudden death testing and observe $Y_{j}$, the time to the first failure from the $\mathrm{j}^{\text {th }}$ group $(\mathrm{j}=1,2, \ldots, \mathrm{g})$. Then calculate the quantity $v_{i+1}=\sum_{j=1}^{g} Y_{j}^{m}$.

- Accept the lot if $v_{i}+v_{i+1} \geq k_{t} L^{m}$ and otherwise reject the lot.

\section{Conditional Double SAmpling Plan Under WEIBULL DisTRIBUTION}

Suppose that the life time of a product follows a Weibull distribution with shape parameter $\mathrm{m}$, and a scale parameter $\delta$ and the cumulative distribution is given by

$$
F(x)=1-\exp \left(-(\delta x)^{m}\right), \quad x \geq 0
$$

Assuming that there is a lower specification limit $L$ regarding the lifetime of the product is given and the shape parameter $\mathrm{m}$ is known, the fraction nonconforming or unreliability is determined by

$$
p=P(X<L)=F(L)
$$

where $X$ is the life time of a product which follows Weibull distribution. If $\mathrm{p}$ is given then $\delta \mathrm{L}$ is obtained from (2) through

$$
w=(\delta L)^{m}=-\operatorname{In}(1-p)
$$

Based on the operating procedure of the proposed conditional double sampling plan, it is to be observed that the acceptance criteria do not involve the scale parameter of the Weibull distribution. The probability of acceptance of the lot based on the sample results of the $i^{\text {th }}$ lot is given by

$$
P_{a i}(p)=1-G_{2 g_{1}}\left(2 r k_{a} w\right)
$$

Where $w$ is given in (3), $G_{\varphi}$ is the distribution function of a Chi-square random variable with $\varphi$ degrees of freedom. The probability of acceptance of the lot based on the sample results of the $(\mathrm{i}+1)^{\mathrm{st}}$ lot is given by

$$
P_{a(i+1)}(p)=P\left\{k_{r} L^{m} \leq v_{i} \leq k_{a} L^{m}, v_{i}+v_{i+1} \geq k_{t} L^{m} \mid p\right\}
$$

Since $2 \delta^{m} r v_{i}$ and $2 \delta^{m} r v_{i+1}$ are independent and follow Chisquare distribution with $2 \mathrm{~g}_{1}$ and $2 \mathrm{~g}_{2}$ degrees of freedom respectively. Now equation (5) reduces to

$$
P_{a(i+1)}(p)=\int\left(1-G_{2 g_{2}}\left(2 r k_{t} w-x\right)\right) \frac{(x / 2)^{g_{1}-1}}{\sqrt{\left(g_{1}\right)}}\left(\frac{1}{2}\right) e^{-\frac{x}{2}} d x
$$

Then the probability of acceptance based on conditional double sampling plan is

$$
L(p)=P_{a i}(p)+P_{a(i+1)}(p)
$$

To determine the optimal parameters of the proposed conditional double sampling plan namely $\mathrm{g}_{1}, \mathrm{~g}_{2}, \mathrm{k}_{\mathrm{a}}, \mathrm{k}_{\mathrm{r}}$ and $\mathrm{k}_{\mathrm{t}}$ the two points on the OC curve approach can be utilized. The probability of acceptance should be atleast $(1-\alpha)$ at the acceptable reliability level $\left(\mathrm{p}_{1}\right)$ and the probability of acceptance should be $\beta$ at the lot tolerance reliability level $\left(\mathrm{p}_{2}\right)$. To obtain the optimal parameters we need to solve the following two equations.

$$
\begin{aligned}
& L\left(p_{1}\right) \geq 1-\alpha \\
& L\left(p_{2}\right) \leq \beta
\end{aligned}
$$

Where $\mathrm{p}_{1}$ is the acceptable reliability level, $\mathrm{p}_{2}$ is the lot tolerance reliability level, $\alpha$ is the producer's risk and $\beta$ is called the consumer's risk. By solving the equation (8), we can get the optimal parameters of the proposed plan and are 
tabulated in Table 1. In this table, we have assumed that $\mathrm{k}_{\mathrm{t}}=\mathrm{k}_{\mathrm{a}}$ in order to reduce the number of parameters.

Table 1: Optimal Parameters of Contional Double Sampling Plan

\begin{tabular}{|c|c|c|c|c|}
\hline & & \multicolumn{3}{|c|}{ Optimal Parameters } \\
\cline { 2 - 5 } $\mathrm{p}_{1}$ & $\mathrm{p}_{2}$ & $\mathrm{~g}$ & $\mathrm{rk}_{\mathrm{a}}$ & $\mathrm{rk}_{\mathrm{r}}$ \\
\hline 0.001 & 0.002 & 18.70 & 12195.0 & 999.9 \\
& 0.004 & 5.10 & 2022.8 & 99.8 \\
& 0.005 & 3.90 & 2000.0 & 100.0 \\
& 0.01 & 2.10 & 404.9 & 0.0 \\
& 0.05 & 1.00 & 300.0 & 5.0 \\
& 0.10 & 0.80 & 200.0 & 5.0 \\
& & & & \\
0.005 & 0.01 & 18.60 & 2417.3 & 500.0 \\
& 0.015 & 7.70 & 757.5 & 2.7 \\
& 0.02 & 5.10 & 398.4 & 0.3 \\
& 0.025 & 3.90 & 256.5 & 0.1 \\
& 0.05 & 2.10 & 78.5 & 0.0 \\
& 0.25 & 0.90 & 26.2 & 0.0 \\
& & & & \\
& 0.02 & 18.50 & 1195.1 & 100.1 \\
& 0.04 & 5.00 & 195.6 & 1.4 \\
& 0.05 & 3.80 & 125.5 & 0.0 \\
& 0.10 & 2.10 & 37.8 & 0.0 \\
& 0.15 & 1.60 & 23.9 & 0.0 \\
& 0.30 & 1.10 & 8.9 & 5.0 \\
& & & & \\
& 0.10 & 17.40 & 217.6 & 50.0 \\
& 0.20 & 4.60 & 33.4 & 0.0 \\
& 0.25 & 3.50 & 20.7 & 0.0 \\
& 0.30 & 2.80 & 14.3 & 0.0 \\
& 0.50 & 1.80 & 5.5 & 0.4 \\
& & & & \\
& 0.20 & 16.10 & 95.8 & 10.0 \\
& 0.40 & 4.00 & 13.2 & 0.0 \\
& 0.50 & 3.00 & 7.6 & 0.0 \\
\hline \multirow{7}{*}{0.10}
\end{tabular}

Example: Suppose that one wants to find a conditional double sampling for given conditions of $\mathrm{p}_{1}=0.01, \mathrm{p}_{2}=0.04$, $\alpha=0.05$ and $\beta=0.10$, Table 1 gives the optimal parameters of the conditional double sampling as $\mathrm{g}=5.00, \mathrm{rk}_{\mathrm{a}}=195.6$ and $\mathrm{rk}_{\mathrm{r}}=1.4$. For the case of $\mathrm{r}=10$, we get the acceptance criterion as $k_{\mathrm{a}}=19.56$ and $\mathrm{k}_{\mathrm{r}}=0.14$. For this example, the proposed plan can be operated as follows.

a. From the lot i, draw a random sample of size $\mathrm{n}=\mathrm{rg}=50$ and allocate 10 parts to each of 5 groups.

- Perform sudden death testing and observe $Y_{j}$, the time to the first failure from the $\mathrm{j}^{\text {th }}$ group $(\mathrm{j}=1,2, \ldots, 5)$. Then calculate the quantity $v_{i}=\sum_{j=1}^{5} Y_{j}^{m}$.

- Accept the lot if $v_{i} \geq 19.56 L^{m}$ and reject the lot if $v_{i}<0.14 L^{m}$. If $0.14 L^{m} \leq v_{i}<19.56 L^{m}$ then defer the decision until the results of the lot (i+1) are obtained.

- From the lot (i+1), draw a random sample of size $\mathrm{n}=\mathrm{rg}=50$ and allocate 10 parts to each of 5 groups.
- Perform sudden death testing and observe $Y_{j}$, the time to the first failure from the $\mathrm{j}^{\text {th }}$ group $(\mathrm{j}=1,2, \ldots, 5)$. Then calculate the quantity $v_{i+1}=\sum_{j=1}^{5} Y_{j}{ }^{m}$.

- Accept the lot if $v_{i}+v_{i+1} \geq 19.56 L^{m}$ and otherwise reject the lot.

\section{CONCLUSIONS}

In this paper, a conditional double sampling plan has been proposed for variables inspection for deciding whether the lot of manufactured products to be accepted or not under sudden death testing. The sudden death testing can be applied to reduce testing time. Tables have also been developed and examples have also been given for implementation of the proposed plan.

\section{ACKNOWLEDGMENT}

The authors would like to thank the editor and the anonymous referees for their useful comments.

\section{REFERENCES}

[1] Baker, R. C. and Brobst, R. W," Conditional Double Sampling”, Journal of Quality Technology, Vol.10, Pp.150-154, 1978

[2] Balamurali, S., Park, H., Jun, C.-H., Kim, K. J. and Lee,J," Designing of Variables Repetitive Group Sampling Plan Involving Minimum Average Sample Number", Communications in Statistics- Simulation and Computation, Vol.34, Pp.799-809, 2005.

[3] Balamurali, S. and Jun, C.-H ,"Repetitive Group Sampling Procedure for Variable Inspection", Journal of Applied Statistics, Vol.33, No.3, Pp.327-328, 2006.

[4] Balamurali, S. and Jun, C.-H," Multiple Dependent State Sampling Plans for Lot Acceptance Based on Measurement Data", European Journal of Operational Research, Vol.180, Pp.1221-1230, 2007

[5] Balamurali, S. and Jun, C.-H,"Designing of a Variables Two-Plan System by Minimizing the Average Sample Number", Journal of Applied Statistics, Vol.36, No.10, Pp.1159-1172, 2009.

[6] Balasooriya, U,"Failure Censored Reliability Sampling Plans for the Exponential Distribution", Journal of Statistical Computation and Simulation, Vol.52, No.4, Pp.337-349, 1995

[7] Govindaraju, K. and Balamurali, S,"Chain Sampling Plan for Variables Inspection", Journal of Applied Statistics, Vol.25, Pp.103-109, 1998

[8] Hamaker, H. C,"Acceptance Sampling for Percent Defective by Variables and by Attributes", Journal of Quality Technology, Vol.11, Pp.139-148, 1979

[9] Jun, C-H, Balamurali, S and Lee, S-H,"Variables Sampling Plans for Weibull Distributed Lifetimes under Sudden Death Testing", IEEE Transactions on Reliability, Vol.55, No.1, Pp.53-58, 2006

[10] Pascaul, F. G. and Meeker, W. Q,'The Modified Sudden Death Test: Planning Life Tests with a Limited Number of Test Portions", Journal of Testing and Evaluation, Vol.26, No.5, Pp.434-443, 1998

[11] Pearn, W.L. and Wu, C.-W,"Critical Acceptance Values and Sample Sizes of a Variables Sampling Plan for Very Low Fraction of Defectives", Omega, Vol.34, Pp.90-101, 2006

[12] Pearn, W.L. and Wu, C.-W,"An Effective Decision Making Method for Product Acceptance", Omega, Vol.35, Pp.12-21, 2007

[13] Sommers, D. J,"Two-Point Double Variables Sampling Plans", Journal of Quality Technology, Vol.13, Pp.25-30, 1981

[14] Vlcek, B. L., Hendricks, R. C. and Zaretsky, E. V:Monte Carlo Simulation of Sudden Death Bearing Testing, NASA, Hanover, MD, 2003

[15] Wu, J.W., Tsai, T. R. and Ouyang, L. Y,"Limited Failure Censored Life Test for the Weibull Distribution", IEEE Transactions on Reliability, Vol.50, N0.1, Pp.107-111, 2001 


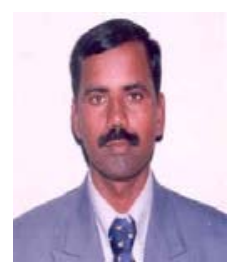

Dr.S. Balamurali is the Professor and Head of the Department of Computer Applications, Kalasalingam University, India. He received his M.Sc. and Ph.D. degrees in Statistics from Bharathiar University, India. His research interests include statistical process control, acceptance sampling and analysis of means. He has so far published more than 50 research papers in reputed National and International journals. His publications have appeared in engineering and applied statistics journals

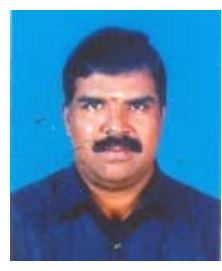

Dr.J. Subramani has received the doctorate degree in Statistics from University of Madras. He is currently working as an Associate Professor, Department of Statistics, Pondicherry University. He has more than 25 years of experience both in Teaching and Research. To his academic credit he has received U.S.Nair Young Statistician Award from Indian Society of Probability and Statistics and also the International Young Statistician Award from the International Statistical Institute, The Netherlands. His research interests are Estimation of Variance Components, Missing Data Analysis, Sampling Theory, Incomplete Block Designs, Control Charts and Process Capability Analysis. He has published more than 75 research papers in reputed International and National Journals. $\mathrm{He}$ has also participated in many conferences and workshops in India as well as abroad and presented research papers; delivered invited talks and special addresses. He has organized many International and National Conferences and also acted as an organizing committee member in many conferences. 